\title{
A computational analysis of heat transfer and fluid flow in high-speed scanning of laser micro-welding
}

\begin{abstract}
A transient three-dimensional model is numerically developed using computational fluid dynamics (CFD) method to understand some critical criteria such as temperature fields and melt pool formation by considering the heat source and the material interaction and the effect of laser welding parameters on laser micro-welding process. To gain more implicit insight of fluid dynamics, the issue of circulation of molten metal assisted by the surface tension, buoyancy and recoil pressure forces in the weld pool has been investigated Assuming that atmospheric

and vaporised material pressures are balanced at the front of the laser beam. The governing equations from the Navier-Stokes for Newtonian fluid are prepared to estimate the melt flow that influences the rate of temperature distribution in a 3-D domain. The simulation results have been compared with two sets of experimental research to predict the weld bead geometry and solidification pattern which laser welds are made on thin stainless steel sheet (SUS304). The shape comparison describes those parameters relevant to any changes in the melt dynamics and temperatures are of great importance on the formation of weld pool and heat distribution during laser micro-welding. The fair agreement between simulated and experimental results, demonstrates the reliability of the computed model.
\end{abstract}

Keyword: Laser micro-welding; Computational fluid dynamics (CFD); Heat transfer and fluid flow analysis 